\title{
Tunnel Vision in a Patient with Polymyalgia Rheumatica
}

MARCIN MILCHERT (D), MD, PhD; MAREK BRZOSKO ¿ , MD, PhD, Professor, Department of Rheumatology, Internal Medicine, Geriatrics and Clinical Immunology of Pomeranian Medical University in Szczecin, Poland. Address correspondence to Dr. M. Milchert, Pomeranian Medical University, Department of Rheumatology, Internal Medicine, Geriatrics and Clinical Immunology, ul. Unii Lubelskiej 1, 71-252 Szczecin, Poland.

E-mail: marcmilc@hotmail.com. Ethical approval was not required by the authors' institution. The patient gave written informed consent to publish the material. J Rheumatol 2020;47:155-6; doi:10.3899/jrheum.190150

Silent vasculitis may accompany polymyalgia rheumatica (PMR), resulting in complications such as vision deterioration. Reduced visual field is a possible manifestation.

A 66-year-old man was diagnosed with PMR and treated accordingly (prednisone maximum $15 \mathrm{mg}$ /day) at a tertiary center, with resolution of his symptoms. After 18 months, he suddenly experienced right eye vision loss. Visual field of his left eye was peripherally limited (Figure 1). Color Doppler ultrasound of proximal part of temporal arteries revealed halo sign consistent with giant cell arteritis (GCA; Figure 2), and stenotic distal branches with decreased flow velocity. High-dose corticosteroids (methylprednisolone $500 \mathrm{mg}$ /day for 3 days) were immediately introduced. The patient regained central vision in his right eye with minor improvement contralaterally. A subsequently acquired distal branch temporal artery biopsy confirmed only fibrosis of the vessel wall. The patient remains stable after
7 years of followup. He adapted well for his disability. While working as a lecturer, he is able to see a single student at the back of an auditorium with his tunnel vision; however, he admits to having problems in climbing a rostrum because of stumbling over the stairs. Low doses of corticosteroids may not suppress silent GCA, leading to temporal artery fibrosis as well as ocular artery branches involvement.

Retrospective analysis revealed that the patient had signs of temporal pain at his first presentation that were masked by dominating PMR manifestations. His GCA might have progressed silently, while undertreated with small corticosteroid doses that resolved PMR manifestations but were not suppressing vascular inflammation, leading to temporal artery fibrosis and vision involvement. Fast-track ultrasound GCA clinics may reduce the rate of sight loss among patients with $\mathrm{GCA}^{1}$.

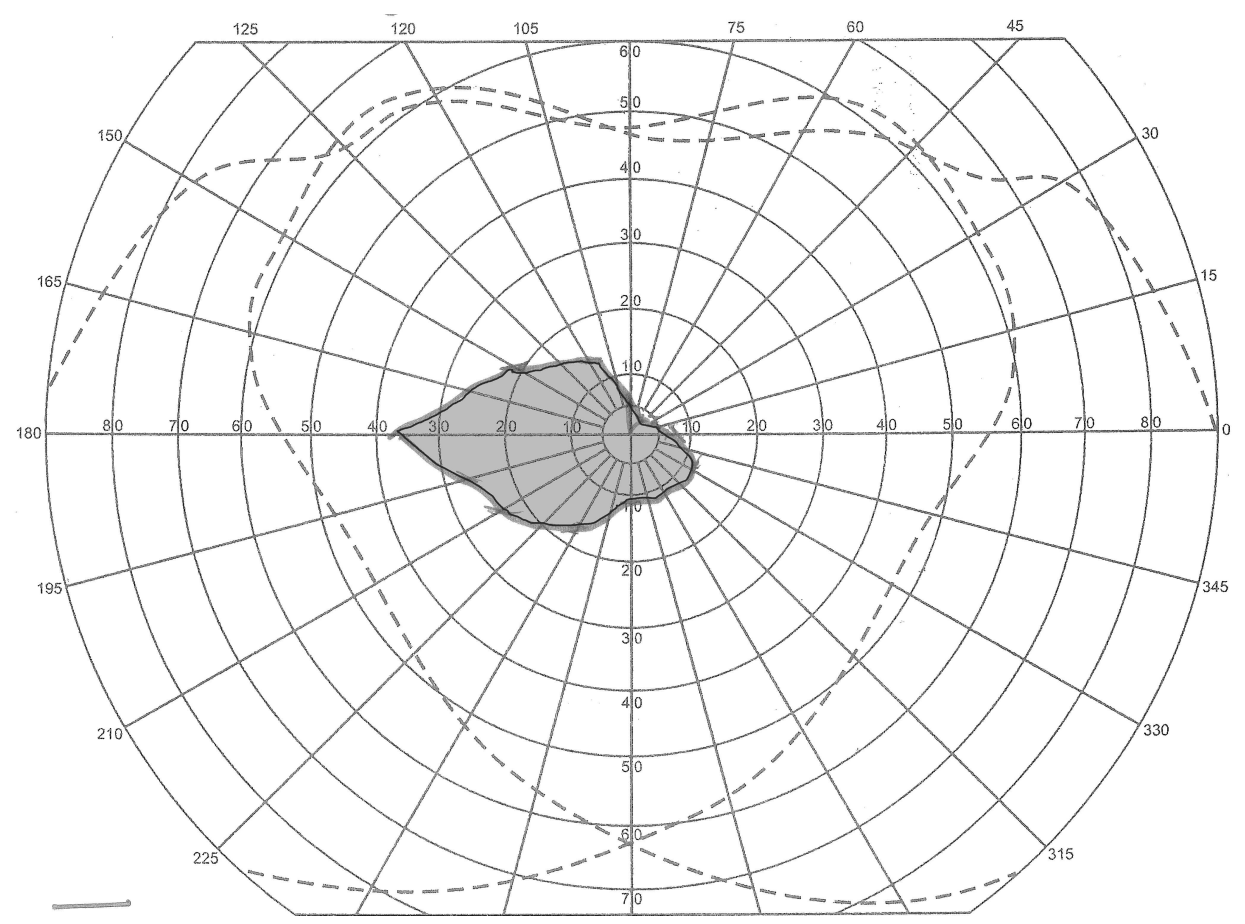

Figure 1. Kinetic perimetry revealing reduced visual field, clinically resulting in tunnel vision. 


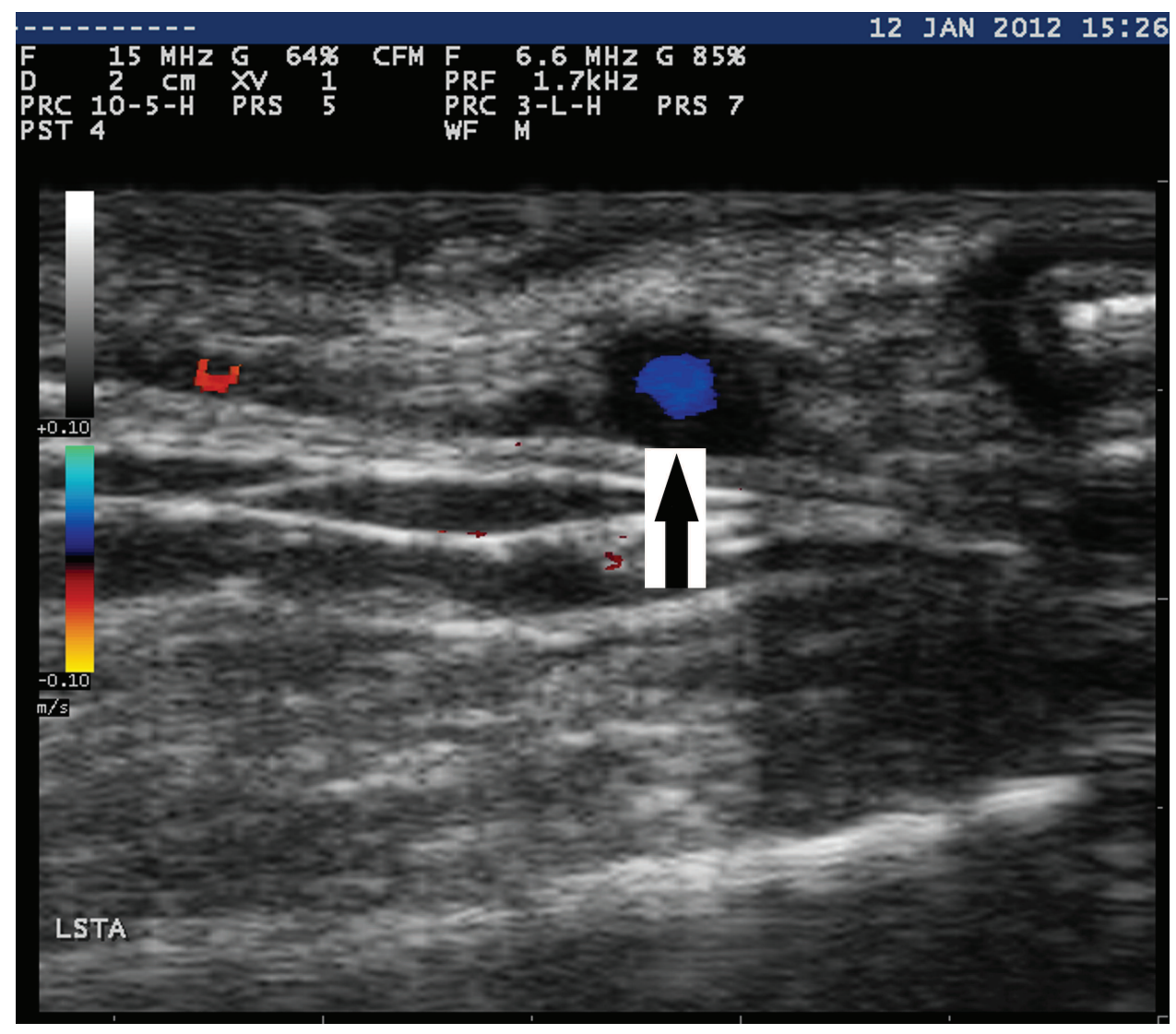

Figure 2. Color Doppler ultrasound of left temporal artery. Halo sign (arrow) visible as hypoechoic, homogeneous concentric thickening of vessel wall in transverse plane, well delineated toward the luminal side, filled with color, confirming partially preserved flow.

\section{REFERENCE}

1. Milchert M, Diamantopoulos A, Brzosko M. Atlas of ultrasound application in large vessel arteritis: giant cell arteritis and Takayasu arteritis. Szczecin: Wydawnictwo Pomorskiej Akademii Medycznej; 2016. 